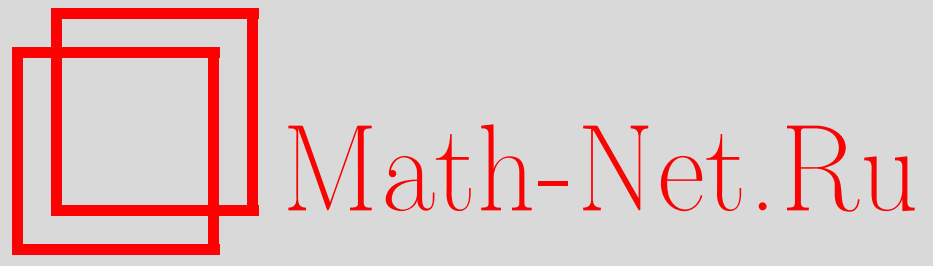

Обцероссийский математический портал

А. В. Иванов, Нульмерное счетно компактное пространство, уплотняющееся на связный бикомпакт, Матем. заметки, 2000, том 68, выпуск 1, 143-145

DOI: https://doi.org/10.4213/mzm929

Использование Общероссийского математического портала Math-Net.Ru подразумевает, что вы прочитали и согласны с пользовательским соглашением http://www.mathnet.ru/rus/agreement

Параметры загрузки:

IP: 3.80 .253 .173

26 апреля 2023 г., 15:24:44 


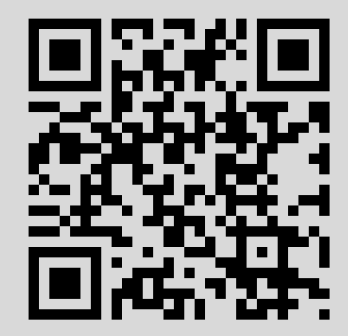




\section{НУЛЬМЕРНОЕ СЧЕТНО КОМПАКТНОЕ ПРОСТРАНСТВО, УПЛОТНЯЮЩЕЕСЯ НА СВЯЗНЫЙ БИКОМПАКТ}

\section{А. В. Иванов}

Широкому обсуждению проблемы уплотнения счетно компактных пространств на бикомпакты посвящена работа А. В. Архангельского и В. В. Федорчука [1]. В ней, в частности, поставлен вопрос о существовании нульмерного счетно компактного пространства, которое уплотняется на связньй бикомпакт (вопрос 5). В настоящей работе этот вопрос решен положительно, а именно, построен пример счетно компактного индуктивно нульмерного пространства $X$ с первой аксиомой счетности, которое уплотняется на связный упорядоченньй бикомпакт $Z$ веса $\omega_{1}$.

Искомый бикомпакт $Z$ мы получим как предел непрерьвного вполне упорядоченного обратного спектра $S=\left\{X_{\alpha}, p_{\beta}^{\alpha}: \alpha, \beta<\omega_{1}\right\}$ длины $\omega_{1}$ из метризуемых упорядоченных компактов с вполне замкнутыми соседними проекциями.

Пусть $X_{0}$ - отрезок $[0,1]$ с обычной интервальной топологией и $A_{0}$ - счетное всюду плотное множество в $X_{0}$. Для каждой точки $x \in A_{0}$ определим непрерывное отображение $h_{x}:[0,1] \backslash\{x\} \rightarrow$ $[0,1]$ по формуле: $h_{x}(t)=0$ при $t<x, h_{x}(t)=1$ при $t>x$. Далее, положим $X_{1}=B\left(X_{0}, Y_{x}, h_{x}:\right.$ $\left.x \in A_{0}\right)$, где $B(\ldots)$ - пространство Федорчука (см. [2]), а $Y_{x}=[0,1]_{x}\left(x \in A_{0}\right)$ - экземпляр отрезка $[0,1]$. В качестве отображения $p_{0}^{1}: X_{1} \rightarrow X_{0}$ возьмем каноническую проекцию $\pi$ пространства $B=X_{1}$ на $X_{0}$. Известно, что отображение $\pi$ вполне замкнуто (см. [2]). Отметим, что в силу выбора отображений $h_{x}$ пространство $X_{1}$ является лексикографическим произведением $X_{0} \bigotimes_{l}\left\{Y_{x}: x \in X_{0}\right\}$ (см. [2]) компакта $X_{0}$ на семейство упорядоченных компактов $\left\{Y_{x}: x \in X_{0}\right\}$, где $Y_{x}=\{\cdot\}$ при $x \notin A_{0}$. Таким образом, $X$ является упорядоченным компактом, который, как легко видеть, связен.

Пространство $X_{1}$ имеет счетную базу, поскольку $A_{0}$ счетно. Множество

$$
C_{1}=\left(p_{0}^{1}\right)^{-1} A_{0}=\bigcup_{x \in A_{0}} Y_{x}
$$

всюду плотно в $X_{1}$. Возьмем в $C_{1}$ счетное всюду плотное множество $A_{1}$ так, чтобы $A_{1}$ не содержало концевых точек каждого отрезка $[0,1]_{x}=Y_{x} \subset C_{1}$, но обязательно содержало его середину. Заметим, что $p_{0}^{1} A_{1}=A_{0}$.

Пусть $\alpha$ - некоторый счетный ординал. Предположим, что для всех $\beta<\alpha$ уже построены пространства $X_{\beta}$, отображения $p_{\gamma}^{\beta}: X_{\beta} \rightarrow X_{\gamma}, \gamma<\beta<\alpha$, и счетные всюду плотные множества $A_{\beta} \subset X_{\beta}$ так, что вьполняются следующие условия. 
$\left.1_{\alpha}\right) X_{\beta}$ - связные упорядоченные компакты, $\beta<\alpha$.

$2_{\alpha}$ ) Спектр $S_{\alpha}=\left\{X_{\beta}, p_{\gamma}^{\beta}: \beta, \gamma<\alpha\right\}$ непрерьвен.

$3_{\alpha}$ ) Отображения вида $p_{\beta}^{\beta+1}$ являются каноническими вполне замкнутыми проекциями лексикографического произведения

$$
X_{\beta+1}=X_{\beta} \bigotimes_{l}\left\{Y_{x}: x \in X_{\beta}\right\}
$$

на компакт $X_{\beta}$, где $Y_{x}=[0,1]_{x}$ при $x \in A_{\beta}$ и $Y_{x}=\{\cdot\}$ при $x \notin A_{\beta}$.

$\left.4_{\alpha}\right) p_{\gamma}^{\beta}\left(A_{\beta}\right)=A_{\gamma}(\gamma<\beta<\alpha)$.

$5_{\alpha}$ ) Множество $A_{\beta+1}$ содержит середину каждого отрезка $\left(p_{\beta}^{\beta+1}\right)^{-1} x=[0,1]_{x}\left(x \in A_{\beta}\right)$ и не содержит его концевых точек $(\beta+1<\alpha)$.

Для формулировки условия $6 \alpha$ ) введем следующее техническое понятие отмеченной точки. Пусть $\beta$ - предельный ординал и $\beta<\alpha$. Точку $x_{\beta} \in X_{\beta}$ будем назьвать отмеченной, если для любого $\gamma<\beta p_{\gamma}^{\beta}\left(x_{\beta}\right) \in A_{\gamma}$ существует $\delta<\beta$ такое, что для любого $\gamma$, удовлетворяющего неравенствам $\delta \leqslant \gamma<\beta$, точка $p_{\gamma+1}^{\beta}\left(x_{\beta}\right)$ является серединой отрезка

$$
\left(p_{\gamma}^{\gamma+1}\right)^{-1}\left(p_{\gamma}^{\beta}\left(x_{\beta}\right)\right)=[0,1]_{p_{\gamma}^{\beta}\left(x_{\beta}\right)} .
$$

Множество отмеченных точек $X_{\beta}$ обозначим через $P_{\beta}$. Поскольку все множества $A_{\gamma}, \gamma<\beta$, счетны, $P_{\beta}$ также счетно.

$\left.6_{\alpha}\right) P_{\beta} \subset A_{\beta}$ для любого предельного $\beta<\alpha$.

Если $\alpha$-предельное число, положим $X_{\alpha}=\lim S_{\alpha}$, а проекции $p_{\beta}^{\alpha}$ определим как предельные проекции спектра $S_{\alpha}$. В силу условий $\left.1_{\alpha}\right)-3_{\alpha}$ ) пространство $X_{\alpha}$ является связным метризуемым упорядоченным компактом (см. [2]).

Пусть $P_{\alpha}$ - множество отмеченных точек $X_{\alpha}$. Покажем, что $P_{\alpha}$ всюду плотно в $X_{\alpha}$. Пусть $\left(x_{\alpha}, y_{\alpha}\right)$ - порядковый интервал в $X_{\alpha}\left(x_{\alpha}<y_{\alpha}\right)$. Существует $\beta<\alpha$ такое, что $p_{\beta}^{\alpha}\left(x_{\alpha}\right) \neq p_{\beta}^{\alpha}\left(y_{\alpha}\right)$, и тогда в интервале $\left(p_{\beta}^{\alpha}\left(x_{\alpha}\right), p_{\beta}^{\alpha}\left(y_{\alpha}\right)\right)$ найдется точка $z_{\beta} \in A_{\beta}$. В силу определения отмеченной точки и выполнения условий $\left.\left.5_{\alpha}\right), 6_{\alpha}\right)$ в пространстве $X_{\alpha}$ найдется единственная отмеченная точка $z_{\alpha}$, для которой $p_{\beta}^{\alpha}\left(z_{\alpha}\right)=z_{\beta}$ (эта точка $z_{\alpha}$ - нить спектра $S_{\alpha}$-однозначно строится по рекурсии). Тогда $x_{\alpha}<z_{\alpha}<y_{\alpha}$.

Итак, $P_{\alpha}$ всюду плотно в $X_{\alpha}$. Положим $A_{\alpha}=P_{\alpha}$. Очевидно, что условия $\left.\left.4_{\alpha+1}\right)-6_{\alpha+1}\right)$ при этом вьполнены.

Пусть теперь $\alpha=\gamma+1$. В этом случае построение пространства $X_{\alpha}$, отображения $p_{\gamma}^{\alpha}$ и счетного всюду плотного множества $A_{\alpha} \subset X_{\alpha}$ проведем по схеме, использованной вьше при построении $X_{1}, p_{0}^{1}$ и $A_{1}$, с той лишш разницей, что вместо $X_{0}$ возьмем $X_{\gamma}$, а вместо $A_{0}-A_{\gamma}$. Связность $X_{\alpha}$ вытекает из связности $X_{\gamma}$ и монотонности $p_{\gamma}^{\alpha}$. Проверка остальных условий $\left.\left.1_{\alpha+1}\right)-6_{\alpha+1}\right)$ тривиальна.

Продолжая рекурсивное построение, мы получим спектр

$$
S=\left\{X_{\alpha}, p_{\beta}^{\alpha}: \alpha, \beta<\omega_{1}\right\},
$$

удовлетворяющий условиям $\left.\left.1_{\omega_{1}}\right)-6 \omega_{1}\right)$. Его предел $\lim S$ является искомым связным упорядоченньм бикомпактом $Z$, имеющим вес $\omega_{1}$.

Обозначим через $P$ множество отмеченных точек $Z$. (На $Z=X_{\omega_{1}}$ определены предельные проекции $p_{\alpha}=p_{\alpha}^{\omega_{1}}: Z \rightarrow X_{\alpha}$ спектра $S$, так что данное выше определение отмеченной точки фактически действует и в $Z$ ). Для любого $\alpha<\omega_{1}$ и любой точки $x_{\alpha} \in A_{\alpha}$ существует единственная точка $z \in P$, для которой $p_{\alpha}(z)=x_{\alpha}$ (как и вьше, точка $z$ однозначно строится по рекурсии). Следовательн, $P$ всюду плотно в $Z$.

Положим

$$
C=\bigcap_{\alpha<\omega_{1}} p_{\alpha}^{-1} A_{\alpha}
$$


Очевидно, что $C \supset P$; следовательно, $C$ также всюду плотно в $Z$.

Для каждой точки $z \in Z \backslash C$ найдется такое $\alpha$, что отображение $p_{\alpha}: Z \rightarrow X_{\alpha}$ взаимно однозначно в $z$. Следовательно, характер $Z$ во всех точках $z \in Z \backslash C$ счетен.

Докажем теперь, что каждое счетное подмножество $K \subset Z$ имеет предельную точку в $Z \backslash C$. Фиксируем счетное $K \subset Z$ и такой индекс $\alpha<\omega_{1}$, что $p_{\alpha}$ взаимно однозначно отображает $K$ на $K_{\alpha}=p_{\alpha}(K) \subset X_{\alpha}$. Пусть $x_{\alpha}-$ предельная точка $K_{\alpha}$. Если $x_{\alpha} \notin A_{\alpha}$, то прообраз $p_{\alpha}^{-1} x_{\alpha}$ содержит единственную точку, которая и будет искомой предельной точкой $K$, лежащей в $Z \backslash C$. Если же $x_{\alpha} \in A_{\alpha}$, то хотя бы одна из концевых точек отрезка

$$
[0,1]_{x_{\alpha}}=\left(p_{\alpha}^{\alpha+1}\right)^{-1} x_{\alpha}
$$

является предельной для $K_{\alpha+1}=p_{\alpha+1}(K)$. Обозначим эту точку через $x_{\alpha+1}$. В силу условия $\left.5 \omega_{1}\right) x_{\alpha+1} \notin A_{\alpha+1}$. Следовательно, $p_{\alpha+1}^{-1} x_{\alpha+1}$ является искомой предельной точкой $K$.

Усилим теперь топологию $Z$ путем объявления всех точек множества $C$ изолированными точками. В итоге получим искомое пространство $X$, которое уплотняется на $Z$.

Действительно, пространство $X$ счетно компактно, поскольку любое счетное подмножество $K \subset Z$ имеет предельную точку в $Z \backslash C$. $X$ индуктивно нульерно, так как $C$ всюду плотно в упорядоченном бикомпакте $Z$. Наконец, $X$ удовлетворяет первой аксиоме счетности, поскольку во всех точках $z \in Z \backslash C$ характер $Z$ счетен.

ЗАмечАниЕ. Построенньй выше бикомпакт $Z$ является $F$-бикомпактом в терминологии [3].

\section{СПИСОК ЦИТИРОВАННОЙ ЛИТЕРАТУРЫ}

1. А рхангельский А. В., Федорчук В. В. // Фундамент. и прикл. матем. 1995. Т. 1. № 4. С. 871-880. 2. Одинцов А. А. Введение в теорию континуумов. М.: Изд-во МГУ, 1989. 3. Иванов А. В. // Матем. заметки. 1986. Т. 39. № 4. С. 606-611. 